\title{
Estudio del potencial innovador en el turismo de los emprendedores cubanos.
}

Study of the innovative potential in tourism of Cuban entrepreneurs.

Mercedes del Carmen Franco Rodríguez. ${ }^{1}$, Yasser Vázquez Alfonso. ${ }^{2}$ \& Efraín Velasteguí López. $^{3}$

Recibido: 15-03-2017 / Revisado: 10-05-2017 Aceptado: 08-06-2018/ Publicado: 01-07-2018

Abstract.

DOI: https://doi.org/10.33262/cienciadigital.v2i3.148

Within the guidelines of the economic and social policy of Cuba is betting that the tourism activity has an accelerated growth that guarantees sustainability and boosts the economy. Cuba's tourism sector reported increases in the number of visitors, reaching in 2017 the 4,257,750 and generating revenues representing $6.9 \%$ of gross domestic product, in 2016. In this context, the tourist offer of the non-state sector takes center stage in accommodation and gastronomy activities, representing $14.9 \%$. However, there is little information on their management models, possibilities for growth and diversification and the productive linkages that it generates. Hence, the purpose of this research aimed at diagnosing forms of training and advisory services demanded by entrepreneurs in order to strengthen the capacity for innovation, finding new methods and products to compete in the market.

Keywords: Non-State Sector, Tourism, Innovation, Entrepreneurs.

\section{Resumen.}

Dentro de los lineamientos de la política económica y social de Cuba se apuesta por que la actividad turística tenga un crecimiento acelerado que garantice la sostenibilidad y dinamice la economía. El sector turístico cubano ha reportado

\footnotetext{
${ }^{1}$ Universidad de la Habana, Facultad de Turismo. Departamento de Formación Básica, La Habana, Cuba, mercedes.franco@ftur.uh.cu

${ }^{2}$ Universidad de la Habana, Facultad de Turismo. Departamento de Formación Básica, La Habana, Cuba, yasser@ftur.uh.cu

${ }^{3}$ Universidad Técnica de Cotopaxi, Ext. La Maná, Cotopaxi, Ecuador, luis.velastegui7838@utc.edu.ec
} 
incrementos en el número de visitantes, alcanzando en el 2017 los 4257750 y generando ingresos que representan el 6,9\% del Producto Interno Bruto, en el 2016. En este contexto la oferta turística del sector no estatal cobra protagonismo en actividades de alojamiento y gastronomía representando el 14,9\%. Sin embargo existe poca información de sus modelos de gestión, posibilidades de crecimiento y diversificación y los encadenamientos productivos que genera. De ahí la finalidad de esta investigación, encaminada a diagnosticar las formas de capacitación y asesoría que demandan los emprendedores con la finalidad de fortalecer la capacidad de innovación, la búsqueda de nuevos métodos y productos para competir en el mercado.

Palabras Claves: Sector no Estatal, Turismo, Innovación, Emprendedores.

\section{Introducción .}

La nueva política económica y social de Cuba apuesta por que la actividad turística tenga un crecimiento acelerado que garantice la sostenibilidad y dinamice la economía, diversificando los mercados emisores y segmentos de clientes y maximizando ingresos. La economía cubana ha crecido entre 2012-2016 a una tasa promedio inferior al 3\% anual, considerada baja si se pretende enrumbar el país en la senda del desarrollo sostenido. En este contexto el sector turístico cubano ha reportado incrementos en el número de visitantes por año, alcanzando en el 2017 la cifra record de 4257 750, generando 3605,9 millones de cuc de ingresos que representan en la estructura del Producto Interno Bruto, el 6,9\% en el 2016 (tabla 1).

Tabla 1. Ingresos del sector turístico en el periodo 2012-2016 en millones de CUC (a precios constantes de 1997).

\begin{tabular}{|l|c|c|c|c|c|}
\hline Año & $\mathbf{2 0 1 2}$ & $\mathbf{2 0 1 3}$ & $\mathbf{2 0 1 4}$ & $\mathbf{2 0 1 5}$ & $\mathbf{2 0 1 6}$ \\
\hline Ingresos totales & 2971,6 & 2972,8 & 2915,0 & 3303,8 & 3605,9 \\
\hline Ingresos sector estatal & 2325,6 & 2325,1 & 2357,3 & 2600,8 & 2907,1 \\
\hline Ingresos del sector no estatal & 358,3 & 365,0 & 378,9 & 485,2 & 537,3 \\
\hline Ingresos por transporte internacional & 287,7 & 282,7 & 178,8 & 217,8 & 161,5 \\
\hline
\end{tabular}

Fuente: Elaborado a partir de datos de la Oficina Nacional de Estadística e Información (ONEI). Anuario Estadístico de Cuba. Edición 2017

Sin embargo las tasas de ocupación siguen siendo bajas debido a la pronunciada estacionalidad. El crecimiento de los ingresos no ha cumplido con las expectativas que genera la dinámica en el número de visitantes y entre las causas pudieran encontrarse "el todo incluido" que no propicia el aumento de los gastos y el crecimiento del turismo de crucero; estos hechos apuntan a la necesidad de desarrollar actividades extrahoteleras y evaluar el crecimiento intensivo en el número de habitaciones, cuando las tasas de 
ocupación no crecen al ritmo esperado. En esta coyuntura el sector no estatal cubano cobra un mayor protagonismo en actividades de alojamiento, gastronomía y otros servicios como oferta turística complementaria a la estatal.

Aunque la legislación cubana no reconoce la existencia de las pequeñas y medianas empresas, la Comisión Económica para América Latina (CEPAL) y otros organismos regionales consideran en sus clasificaciones de PyMES fundamentalmente dos criterios: la cantidad de empleados y los ingresos que generan. Aunque no sea reconocido formalmente el sector no estatal cubano está integrado mayoritariamente por estas formas organizativas, constituyendo la transformación económica más importante del modelo económico cubano y han ido ganando espacio (figura 1).

Figura 1. Estructura de ingresos del sector turístico cubano en porciento (20122016).

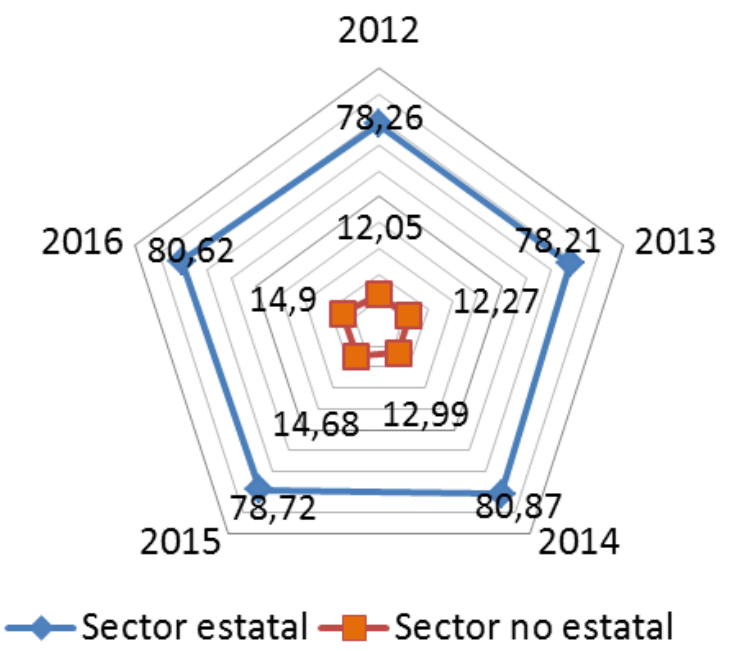

Elaborado por: Grupo de investigación.

En el turismo, las formas de gestión relacionadas con el alojamiento y la gastronomía representaron en el 2016 el 14,9\% del total de ingresos del sector. Este hecho merece ser objeto de estudio ya que estas formas organizativas pueden significar: generación de empleo, encadenamientos productivos, innovación de productos, servicios y formas de comercialización que cobran especial relevancia en el contexto de la economía cubana.

El fortalecimiento del sector no estatal puede contribuir a minimizar impactos negativos del turismo como la fuga de los beneficios debido a que las grandes cadenas trasnacionales, las aerolíneas, o los operadores, radican fuera de los destinos. La apertura de estas formas 
empresariales es una forma de reducir el déficit fiscal y constituyen importantes fuentes de empleo. Generalmente requieren de inversiones menores y pueden lograr una gran flexibilidad para ofertar productos o servicios que cumplan con las expectativas de los consumidores; constituyendo importantes espacios para el desarrollo de habilidades creativas e innovadoras.

Sin embargo el desarrollo de este sector atraviesa por no pocas dificultades; aunque se han dado pasos para facilitar las interrelaciones entre los sectores estatal y no estatal, faltan mecanismos que faciliten la integración entre esos actores. El acceso a los servicios de capacitación y asesoría no ha sido resuelto.

Es conocido que el otorgamiento de créditos para apoyar la actividad no estatal corresponde a la política del estado cubano y se espera que dinamice la actividad bancaria; no obstante la relación de este segmento con la banca cubana es limitada. Desde la entrada en vigor del decreto Ley No. 289, en diciembre del 2011 los bancos que se vincularon a la banca de desarrollo fueron el Banco Metropolitano de la Habana, Banco Popular de Ahorro y Banco de Crédito y Comercio, este último ha logrado financiar a penas el $6 \%$ del mercado potencial del sector no estatal que asciende a 286000 negocios (Castro, 2018) y situaciones similares se registran en las otras entidades bancarias.

Sin embargo, existen señales del sector no estatal como el crecimiento rápido de la oferta en Airbnb ${ }^{4}$ que indican sus potencialidades y capacidad de adaptación a los códigos de comercialización y venta de servicios que rigen el mercado internacional. A pesar de las dificultades de conectividad y las regulaciones que impone una empresa de este tipo; esta plataforma de software tiene a Cuba como uno de los mercados de más rápido crecimiento pasando de 4000 casas de alquiler en el 2015 a 32000 casas en febrero del 2018, generando ingresos por 40 millones de dólares.

Estos elementos apuntan a la existencia de una brecha metodológica importante que merece ser objeto de estudio, las características particulares de la economía cubana y de los Trabajadores por Cuenta Propia (TCP), hacen que estas formas organizativas se distingan de sus similares en América Latina demandando de la academia y de los investigadores de las ciencias económicas una mirada específica.

\section{Marco Teórico Referencial.}

Diversos autores han hecho hincapié en el hecho de que las ventajas competitivas más sólidas nacen esencialmente de la capacidad para innovar, de la búsqueda de nuevos métodos para competir y de mejores medios para luchar por los mercados. El proceso de innovación incluye además de nuevas tecnologías, nuevos métodos, el diseño de nuevos

\footnotetext{
${ }^{4}$ Empresa y plataforma de software cuyo nombre proviene del acrónimo de airbed and breakfast (colchón inflable y desayuno).
} 
productos y procesos de producción, las estrategias de mercadeo, las formas de capacitar al personal y las capacidades para organizar todos los elementos de la actividad empresarial (Micheli, 1993).

Entre las características más importantes de las mipymes latinoamericanas de acuerdo a expertos de la CEPAL (1988) se podrían considerar las siguientes: baja intensidad de capital; altas tasas de natalidad y mortalidad; alta participación de propietarios, socios y miembros de la familia en los negocios; no existe distinción en términos financieros y contables, la persona física de la jurídica; registros contables poco adecuados; contratación directa de mano de obra; utilización de mano de obra poca o no calificada; baja inversión en innovación tecnológica; poco acceso a los mercados internacionales; bajo dominio del inglés y difícil acceso al financiamiento de capital.

Estas características presentan similitudes con las mi pymes de la Unión Europea según Martínez y Lombana (2013) y también diferencias sustanciales relacionadas con la presencia de la informalidad en América Latina, la tecnología, la capacidad de innovación y el acceso al capital. Estas diferencias hacen que las condiciones de competitividad sean diferentes. En el caso cubano según datos oficiales el sector no estatal relacionado con el turismo ha crecido a ritmos discretos, sin embargo su potencial innovador ha sido poco estudiado; la innovación no solo es la aplicación de I+D+i a alto nivel, esta se encuentra relacionada con las capacidades emprendedoras de decisión y organización. En el turismo la inserción de la innovación en productos, procesos, y en la mercadotecnia son imprescindibles como soporte a la competitividad.

Investigaciones desarrolladas por el Centro de Estudios de la Economía Cubana (CEEC) y la Red de Emprendimiento e Innovación de la Universidad de la Habana han permitido ahondar en los factores que obstaculizan e impulsan el emprendimiento en Cuba. Identificando como los principales obstáculos: la ausencia de un mercado mayorista, la forma de regulación de la actividad, la falta de apoyo y educación específica, la burocracia y el limitado acceso financiero. Como principales motores, los investigadores recogen: las necesidades económicas, los políticas públicas, las condiciones del mercado, los factores culturales y el espíritu emprendedor (Díaz, 2017).

No obstante la existencia de estos estudios; existe poca información de cómo realizan su gestión estas entidades del sector no estatal, sus niveles de actividad, empleados, ingresos, posibilidades de crecimiento y diversificación, los encadenamientos productivos que generan y su capacidad de innovación. Uno de los elementos que dificulta las investigaciones es la resistencia de los cuentapropistas a ofrecer información que pueden considerar sensible para el desarrollo de sus negocios. Se puede acceder a los datos de la 
Oficina Nacional de Administración Tributaria (ONAT) no obstante esta información esta sesgada por el hecho de que se recopila con fines fiscales; de ahí la necesidad de desarrollar investigaciones que permitan buscar estrategias para caracterizar el sector no estatal;

- ¿Cómo enfocan su gestión?

- ¿Cómo pueden maximizar sus beneficios?

- ¿Cuáles son sus potencialidades?

- ¿Generan encadenamientos productivos?

- ¿Dinamizan la banca?

- ¿Qué oportunidades tiene el sector no estatal de un mayor protagonismo?

- ¿Cuáles son sus reservas de innovación?

- ¿Qué papel desempeña la universidad en el proceso de desarrollo de este sector?

Cuando se describe en la literatura científica el término emprendedor se hace referencia a la persona que encuentra una oportunidad de negocio y la desarrolla con actitud y aptitud para enfrentar nuevos retos y proyectos. Existe consenso que las características que debe reunir un emprendedor son: creatividad, asunción de riesgos, habilidad para adaptarse a nuevas situaciones, saber priorizar, la perseverancia, su capacidad de comunicar, socializar y adaptarse a las circunstancias.

Las relaciones que se establecen entre la capacidad para emprender e innovar han suscitado interés académico, hacia los factores que propician que algunas empresas sean más innovadoras que otras. La capacidad de innovación de la joven empresa ha sido analizada desde una perspectiva interna considerando características individuales y organizacionales del emprendedor y desde la perspectiva externa que incluye el entorno sectorial y geográfico (González, 2007). En este estudio abordaremos la capacidad de innovación vinculada al capital humano, su edad, nivel de escolaridad y características emprendedoras, aspectos ampliamente abordados en la literatura científica (Hadjimanolis, 2000; Cliff et al., 2005; Levenburg et al.,2006).

La edad es una de las variables que se presume se relaciona con la capacidad de innovación de los emprendedores no obstante los resultados obtenidos en varios estudios difieren significativamente. A priori cabría esperar que un innovador con mayor edad estaría mejor capacitado para innovar, sin embargo con la edad también cambia la propensión a asumir riesgos y reinventarse. En esta investigación defenderemos la hipótesis que la capacidad de innovación del emprendedor, se relaciona con el nivel educativo de manera significativa.

Marco Metodológico. 
Con el objetivo de realizar un acercamiento al perfil del emprendedor cubano dedicado a la actividad de alojamiento se realizó una encuesta. Para realizar el perfil del emprendedor se descartó la realización de un muestreo aleatorio auténtico debido a las características del sector no estatal cubano. Se conoce por estudios realizados con antelación con los arrendatarios cubanos que la obtención de información que pueda considerarse sensible para el sector privado, es difícil.

Tomando en consideración las experiencias de colegas que han incursionado en estas temáticas se decidió aplica un muestreo no probabilístico por Bola de Nieve. La muestra no probabilística no es producto de una selección aleatoria, los elementos son seleccionados en función de su accesibilidad o a criterio personal o intencional del investigador. En el caso que nos ocupa como el segmento que se investiga es de difícil acceso, se contactará intencionalmente con arrendatarios conocidos por el investigador que tengan disposición para ser incorporados al estudio y a partir de ahí, ellos recomendarán a otros arrendatarios predispuestos a cooperar en la investigación.

El uso de este tipo de muestreo implica ventajas; el proceso en cadena permite al investigador llegar a poblaciones de difícil acceso con otros tipos de muestreo, el proceso necesita poca planificación, es simple y rentable. No obstante también implica desventajas: se tiene poco control sobre el muestreo, los sujetos seleccionados dependen de los sujetos observados anteriormente, es probable un sesgo de muestreo ya que los primeros sujetos tienden a designar personas que conocen, lo que hace muy probable que compartan las mismas características. Estas desventajas hacen que los resultados de este estudio se suscriben a la muestra estudiada y no deben ser utilizados en generalizaciones para toda la población.

Con el objetivo de despejar algunas de estas interrogantes se llevó a cabo un estudio de tipo descriptivo, aplicando una encuesta a 128 arrendadores.

\section{Cuestionario para arrendadores}

Estimado arrendador; la Facultad de Turismo de la Universidad de la Habana se encuentra realizando una investigación con vistas a fortalecer la innovación entre los trabajadores por cuenta propia y estrechar los lazos entre la universidad y el sector empresarial. Le

solicitamos amablemente que nos proporcione algunos datos personales de manera totalmente anónima. Agradeciendo anticipadamente su colaboración.

Edad:

Sexo: $F_{-} M_{-}$

Nivel de escolaridad: Primario_Secundario_Preuniversitario_Universitario_

1. Es capaz de comunicarse con sus clientes en:

Inglés_Francés_Italiano_Alemán_Otros_ Ninguno_ 
2. ¿Qué otros servicios además de alojamiento presta usted?

Gastronomía_Lavandería_Transporte_Información_Guía turística_Traductor_ Ninguno_

3. ¿A través de qué vía se promociona su servicio?

Facebook_Plataformas digitales_Tarjetas__Clientes repitentes_

No se realiza promoción_

4. ¿Le gustaría que las agencias cubanas promocionaran su negocio?

Sí_No_

5. Teniendo en cuenta su actitud con el manejo de su negocio, ¿posee usted las características que se exponen a continuación?

Creativo/a_ Buen/a comunicador/a_ Entusiasta__ Dinámico/a_ Competitivo/a_ Innovador/a

6. En el futuro cómo ve su negocio:

Lo dejaría_ Lo mantendría como está_ Quisiera ampliarlo_

Quisiera crear nuevos negocios_ Me gustaría transformarlo_

7. ¿Le gustaría recibir asesoría con vistas a mejorar su negocio?

Sí_ No_

8. ¿En qué temáticas necesitaría capacitarse?

Ninguna_ Administración_ Contabilidad_ Planes de negocios_

Idiomas_C Comercialización_ Computación_ Otras

\section{Análisis de los Resultados.}

Con los resultados de las encuestas aplicadas se realizó el procesamiento en el SPSS 22.0. Se creó un fichero de datos con 11 variables: edad, Sexo, nivel de escolaridad, dominio de otros idiomas, encadenamiento con otros servicio, vías de promoción, necesidad de promoción, perfil del emprendedor, visión de futuro, demanda de capacitación y temática de capacitación.

Participaron un total de 128 arrendadores de la Ciudad de la Habana, de ellos 61 feminas y 67 hombres. Los principales resulatdos obtenidos arrojan que 78 poseen estudios universitarios, lo que representa un $70,23 \%$ de la muestra. 
El $90 \%$ de los arrendatarios manifiesta que es capaz de comunicarse con sus clientes en otros idiomas, y de ellos el $94 \%$ en idioma inglés. Otras lenguas que se dominan frecuentemente son: el francés, el italiano y el un porciento menor el alemán, como se puede apreciar en la figura 2.

Figura 2. Dominio de idiomas extranjeros

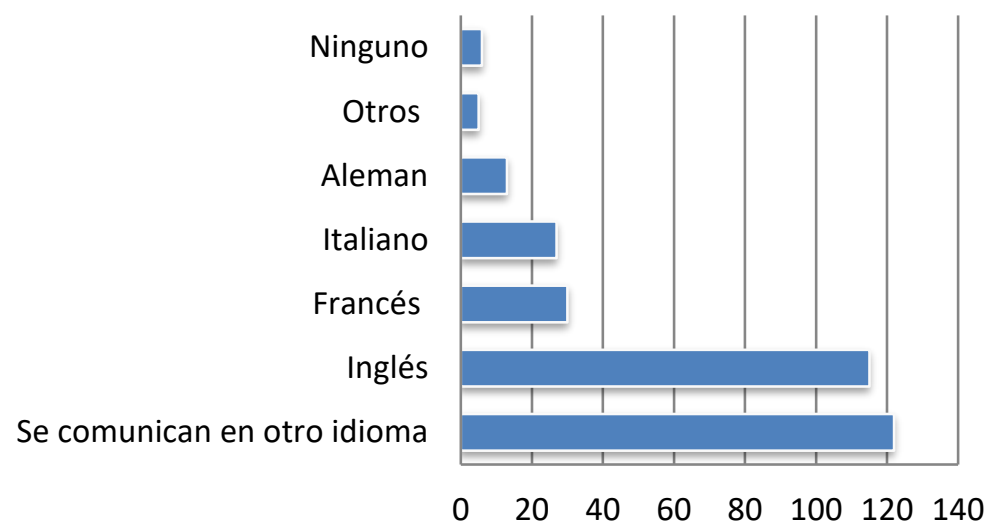

Elaborado por: Grupo de investigación.

Al realizar un análisis del perfil emprendedor de los arrendatarios se aprecia que los mismos se reconocen como buenos comunicadores, creativos y competitivos. No obstante no sucede lo mismo con la capacidad de innovación con la que se identifican solo 63 de los encuestados.

Figura 3. Auto reconocimiento de las habilidades como emprendedor

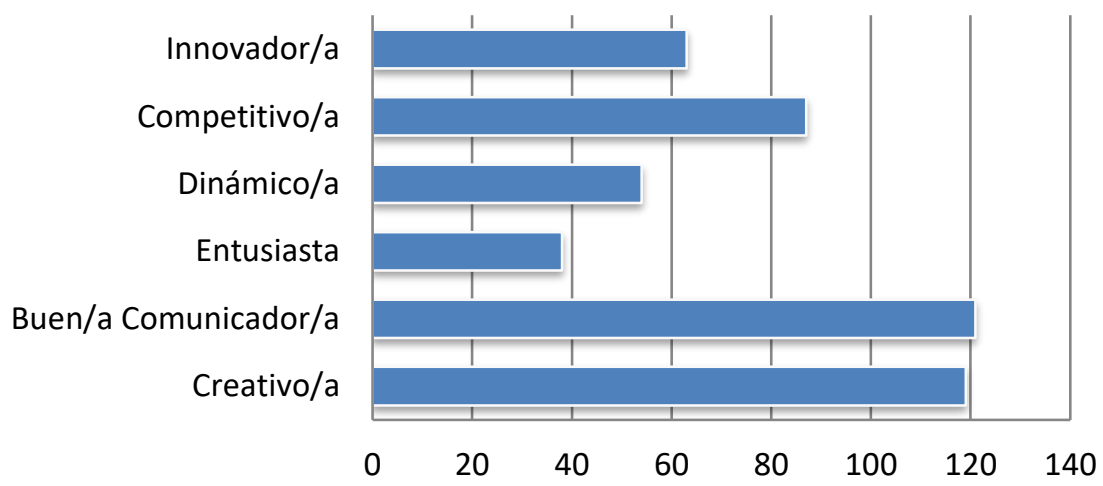

Elaborado por: Grupo de investigación. 
Estos resultados demuestran que los arrendatarios encuestados no se identifican con el término innovación; y si con la competitividad. Esta falta de reconocimiento pudiera estar relacionada con la naturaleza de las innovaciones en el turismo, donde pueden distinguirse por ser radicales o incrementales y relacionarse con productos, servicios o procesos. Sin embargo al ser encuestados sobre el futuro de sus negocios el $62 \%$ manifiesta que quisiera transformarlo, el $34 \%$ ampliarlo y un $27 \%$ quisiera incursionar en nuevos negocios, denotando que no son adversos al riesgo.

Importante para la retroalimentación con la academia resultan los datos relacionados con las necesidades de asesorías y capacitación de los arrendadores. Solo el $2 \%$ de los incluidos en el estudio no desea recibir asesoría de la universidad y el 93\% está interesado en cursar cursos de posgrado; fundamentalmente en diseños de planes de negocios, idioma y comercialización. Estos datos son un diagnóstico inicial sobre la necesidad de capacitación de este segmento y su propensión a ser incluidos en los planes de posgrado universitarios.

\section{Conclusiones.}

- La generación de información sobre los arrendadores es imprescindible para impulsar el emprendimiento en el turismo y genera importantes salidas para los decisores de las políticas públicas y los investigadores del tema.

- El estudio permitió caracterizar el perfil de 128 arrendadores de la Ciudad de la Habana en cuanto a: creatividad, comunicación, entusiasmo, dinamismo, competitividad e innovación; así como su visión de futuro del negocio. A la postre estos elementos permitirán distinguir las características propias del ecosistema emprendedor cubano, que a juzgar por el dominio de idiomas y el nivel de escolaridad de los arrendatarios difiere en esos aspectos de los latinoamericanos.

- La falta de educación específica dirigida desde la universidad a los TCP merece especial atención, pues los resultados demuestran que existe una demanda de este segmento de asesorías y cursos relacionados fundamentalmente con el diseño de planes de negocios, idiomas y la comercialización.

- La realización de investigaciones es este segmente de TCP contribuirá a incrementar la competitividad de Cuba en los mercados turísticos, fomentando la capacitación de los recursos humanos, la calidad de los servicios y la innovación en las formas de gestión y comercialización.

\section{Referencias bibliográficas.}

Castro Morales, Yudy (2018). "De fiadores ingenuos y otras garantías". Periódico Granma. 14 de abril 2018. 
CEPAL. (1988). El proceso de desarrollo de la pequeña y mediana empresa y su papel en el sistema industrial: el caso de Italia. Santiago de Chile: Naciones Unidas.

Díaz Fernández, Ileana; Ferre Menza, Zuleika (2017). “Emprendimiento en Cuba: Criterio de expertos". Miradas a la Economía Cubana. Un acercamiento a la actualización seis años después. Colectivo de autores del Centro de Estudios de la Economía Cubana. 2017.

Fernández Jardón, C. M. (2012). Determinantes de la capacidad de innovación en PYMES regionales. Revista de Administraçao da Universidade Federal de Santa María, 5.

González, JL; Peña, I (2007) Determinantes de la capacidad de innovación de los negocios emprendedores en España.

Martínez, D .Lombana J (2013). Pymes, Rutas para la Exportación. Barranquilla, Universidad del Norte.

Moreno Rojas, S.I.D.I.A., \& García Carrillo, À. (2014). Sistema para la evaluación de capacidades de innovación en pymes de países en desarrollo: caso Panamá. Revista Facultad de Ciencias Económicas: Investigación y Reflexión, 22(2).

Oficina Nacional de Estadística e Información (2017). Anuario Estadístico de Cuba. Edición 2017. Capítulo 5. Cuentas Nacionales.

Pizzi, A., \& Brunet, I. (2013). Creación de empresas, modelos de innovación y pymes. Cuadernos del CENDES, 30(83), 53-74.

Ripoll, F. S., \& Hervás-Oliver, J. L. (2011). ¿ Qué explica la innovación en PyMEs?. Dirección y Organización, (43), 5-15.

Rodeiro Pazos, D., \& López Penabad, M. C. (2007). La innovación como factor clave en la competitividad empresarial: un estudio empírico en pymes. Revista galega de economía, 16(2).

Silveira-Pérez, Y., Cabeza-Pullés, D., \& Fernandez-Perez, V. (2016). Entrepreneurship: Cuban perspective on the creation of family businesses. European Res

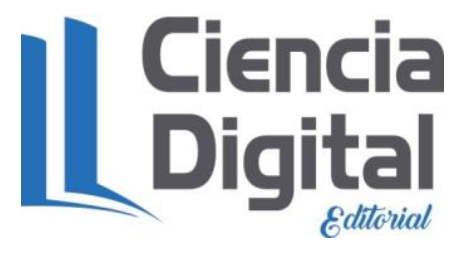




\section{Para citar el artículo indexado.}

Franco M. \& Vázquez Y. . (2018). Estudio del potencial innovador en el turismo de los emprendedores cubanos. Revista electrónica Ciencia Digital 2(3), 216-227. Recuperado desde:

http://cienciadigital.org/revistacienciadigital2/index.php/CienciaDigital/article/view/148/13 $\underline{3}$

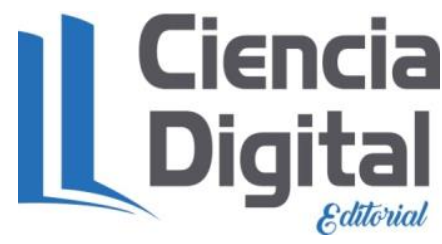

El artículo que se publica es de exclusiva responsabilidad de los autores y no necesariamente reflejan el pensamiento de la Revista Ciencia Digital.

El articulo queda en propiedad de la revista y, por tanto, su publicación parcial y/o total en otro medio tiene que ser autorizado por el director de la Revista Ciencia Digital.
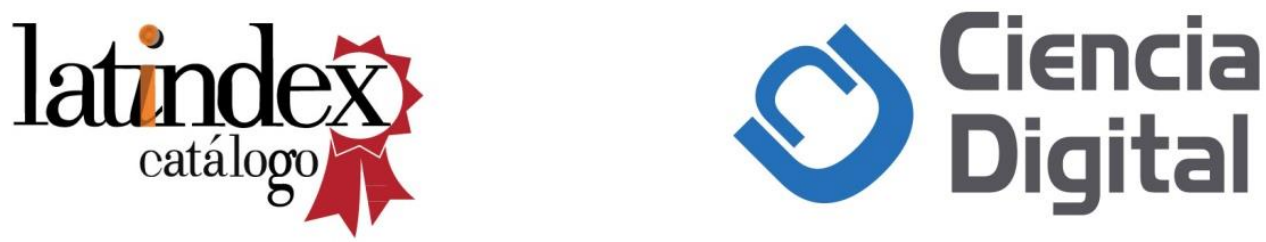